\title{
Data Mining and System Pharmacology to Elucidate the Efficiency and Mechanism of Chinese Medicine in Treating Primary Liver Cancer
}

\section{Zhen Zhang}

Hunan University of Chinese Medicine

\section{Puhua Zeng}

Affiliated hospital of hunan academy of traditional chinese medicine

\section{Wenhui Gao}

Hunan University of Chinese Medicine

\section{Sha Tian}

Hunan University of Chinese Medicine

\section{Qing Zhou}

THE FIRST AFFILIATED HOSPITAL OF HUNAN UNIVERSITY OF CHINESE MEDICINE

\section{Yi Wang}

Hunan University of Chinese Medicine

\section{Wenhao Zhu}

Hunan University of Chinese Medicine

Xuefei Tian ( $\nabla$ txf1718@163.com )

Hunan university of Chinese medicine https://orcid.org/0000-0001-9257-1449

\section{Research}

Keywords: Primary liver cancer, Clinical Research, System Pharmacology, Chinese medicine

Posted Date: November 5th, 2020

DOI: https://doi.org/10.21203/rs.3.rs-101900/v1

License: (c) (1) This work is licensed under a Creative Commons Attribution 4.0 International License. Read Full License 


\section{Abstract}

\section{BACKGROUND}

Piling studies have demonstrated that Chinese medicine (CM) is effective in treating PLC. However, underlying mechanism of $\mathrm{CM}$ in treating PLC remains confused.

\section{MATERIAL AND METHOD}

The information of 524 patients with PLC were collected and separated to CM group and Less-CM group based on the time of taking $\mathrm{CM}$. Then, the prescriptions in $\mathrm{CM}$ group were collected to identify the corn drugs. System Pharmacology was employed to explore the mechanism of corn drugs in treating PLC.

\section{RESULTS}

In univariate and multivariate analysis, taking Chinese medicines more than 6 months was an independent prognostic factor. Then, Traditional Chinese Medicine Inheritance Support System was employed to find out the core drugs that really benefit for PLC patients. Totally, 153 ingredients from 9 core drugs (HXCF) were screened out. Fifty-five genes were identified for HXCF against PLC. The pathways were found as the mechanism of HXCF to treat PLC. Docking studies suggested amount of ingredients in HXCF exerts therapeutic effects against PLC, at least in part, by modulating the function of c-JUN and IL-6.

CONCLUSION

Our results verified the effectiveness of $\mathrm{CM}$ in treating PLC and mined the corn drugs. What's more, our result also predicted active components and potential targets of HXCF for the application to PLC from a holistic perspective, as well as provided valuable direction for further research of HXCF and improved comprehension of PLC.

\section{Introduction}

Primary liver cancer(PLC) has been cursed for human beings for a long time, especially in China, which is related to $1 \%$ of mortality approximately[1]. According to 2018 GLOBALCAN, liver cancer is the fourth leading cause of death and more than 782,000 died due to PLC, worldwide[2].

Currently, concomitant diseases, e.g. chronic hepatitis B or C virus (HBV or HCV), cirrhosis, are the major risk factors for PLC. In China, $80 \%$ to $90 \%$ of PLC is associated with chronic HBV or HCV infection[3,4]. Prevalence closely mirrors its sharply late-stage presentation, absence of specific symptoms, restricted treatment options, aggressive nature and very poor overall survival, which conspire to culminate in a median overall survival of $<6$ months[5]. Thus, annual mortality figures virtually equal to incidence numbers[6]. Thus, a starling lack of effective treatment is still the biggest challenge for PLC. 
Chinese medicine (CM) has been widely utilized to treat disease for thousands of years. However, its clinical efficiency has not been clarified systematically. And the relationship between CM prescription patterns and better survival rates in PLC need further investigation. What's more, the mechanism requires further discussion. Thus, the present study was aimed to explore the efficiency and mechanism of CM in treating PLC.

Huge amount of data with the application of $\mathrm{CM}$ is available to mine the core medicines. Developed by the Institute of Traditional Chinese Medicine of the Chinese Academy of Traditional Chinese Medicine and the Institute of Automation of the Chinese Academy of Sciences, Traditional Chinese Medicine Inheritance Support System(TCMISS) largely concentrate on the fundamental issues about how to expedite its inheritance, development, dissemination and innovation[7]. Supported by the methods and techniques of artificial intelligence, data mining, network science and other disciplines, TCMISS for the inheritance of $\mathrm{CM}$ has effectively solved the problems of non-standardization and personalization in the process of inheritance of $\mathrm{CM}[8-10]$.

Gene Expression Omnibus (GEO) was created by the National Biotechnology Information Center (NBIC) of the United States, containing high-throughput gene microarray and next-generation sequence functional genomics data. Comprehensive bioinformatic analysis of the aberrant gene expression about PLC could lead to better understanding of pathological process of PLC[11].

Clinically, CM was utilized according to patients' syndrome differentiation and viewing the patient as a whole. Therefore, CM to treat PLC doesn't focus on the tumor itself, but on correcting the internal imbalance responsible for tumor development and progression. When prescription is employed, thousands of molecules entry into the human body and perform multiple targets. Therefor it brings great challenges to research $\mathrm{CM}$, precisely. The emergence of system pharmacology brings new hope for research of $\mathrm{CM}$, which can comprehensively elucidate complex relationship between $\mathrm{CM}$ and disease. Up to present, system pharmacology has been successfully employed to explore various CM, Danshen formula in treating cardiovascular disease, Xianlingubao Prescription in treating osteoporosis, Kushen injection in treating gastric cancer, etc[12-14].

In this work, a retrospective study was applied to illustrate whether patients with PLC can benefit from the treatment of CM. Then, TCMISS was utilized to find out core drugs. In addition, differential expressed genes about PLC in the GEO were found out. System pharmacology provides a desirable support to further investigate the mechanism of the core drugs in the treatment of PLC, which provides scientific basis for the CM in treating PLC (as shown in figure 1).

\section{Methods}

1. Patient characteristics.

The patients were selected for our study as following criteria: $18 \leq$ age $\leq 75 \rrbracket$ the total of survival time $\geq 6$ months; diagnosed by pathological examination or clinical diagnosis. The major exclusive criteria as 
follows: concurrent other malignant tumors; comorbidities included severe diseases (severe cardiac

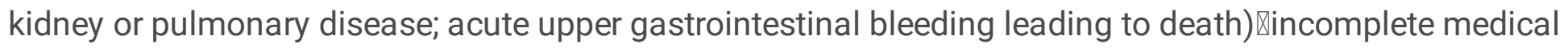
record; loss to follow-up. Informed consent was waived off due to the retrospective design of the study.

\section{Treatment}

All the patients were received routine treatments. Based on their wishes, the patients choose to take CM or not. According to the syndrome differentiation, all the patients were administered individually, wherein the formula was administered orally two times daily 30 minutes after meals.

3. Identification of core drugs

The prescriptions in CM group were collected. From the date of receiving CM treatment to the patient's death or up to the time of the research, the patient prescriptions of CM group were collected. Refer to the 2015 edition of Chinese Pharmacopoeia to unify the name of CM to their official name. According to this criteria, "chaihu(local fried or bran fried)" is equal to "chaihu". "shengshaishen" is dubbed for "renshen". In order to decrease the bias, the CM not included in the Pharmacopoeia, such as "baihuasheshecao", is uniformly named with reference to textbooks or literatures. Then the prescriptions were input TCMISS by professional worker and checked two times by another two workers to assure its correctness, accuracy and reliability. Based on the threshold of "support degree" to 300 and the "confidence level" to 0.95, "Data analysis" module in TCMISS was employed to acquire core drugs (HXCF).

\section{Screening the active ingredients of $\mathrm{HXCF}$}

All of the ingredients in HXCF were retrieved from the relevant database, including Traditional Chinese Medicine Systems Platform (TCMSP, http://tcmspw.com/tcmsp.php, Version: 2.3, accessed March 2020), Natural Product Activity \& Species Source Database(NPASS, http://bidd2.nus.edu.sg/NPASS , accessed March 2020), A Bioinformatics Analysis Tool for Molecular Mechanism of Traditional Chinese Medicine (BATMAN-TCM http://bionet.ncpsb.org/batman-tcm/ , accessed March 2020). Finally, 1125 chemical ingredients were collected. Then, the structure of the chemical ingredients were acquired from NCBI PubChem database (https://www.ncbi.nlm.nih.gov/, accessed March 2020). Then, the ingredients were screened as following criteria.

Oral bioavailability (OB) is named as the relative amount of unmodified drug absorbed into the circulatory system after extravascular administration. It is a significant index to evaluate the efficacy of drug absorption[15]. Components with high OB are often regarded as the potential drugs being developed. Therefore, chemicals with $\mathrm{OB} \geq 30 \%$ were selected as the threshold value for screening potential ingredients.

Drug-Likeness (DL) is a criterion to evaluate the pharmacokinetic characteristics of chemicals in human body, comprehensively[16]. DL is used to filter excellent ingredients with undesirable properties. The DL is calculated as the following formula: 


\section{$D L(X, Y) \frac{X * Y}{|X|^{2}+|Y|^{2}-X * Y}$}

In the formula, $\mathrm{X}$ represents the chemical properties of herbal ingredients based on

Dragon soft molecular descriptors, and $\mathrm{Y}$ represents the average drug-likeness index of all 6511 molecules in Drug-Bank database ( http://www.drugbank.ca/ accessed March 2020)[17]. In this study, the criterion, $\mathrm{DL} \geq 0.18$, was defined to screen out potential active ingredients.

The drug half-life $(\mathrm{HL})$, commonly named as the biological half-life, refers to the time it takes for the concentration of the drug in the blood or the amount of the drug to be decreased to half in the body[18]. In this study, $\mathrm{HL} \geq 4$ was defined as the criteria to select potential active ingredients.

\section{Identification of the chemical targets of HXCF}

The chemical targets of HXCF were acquired from TCMSP database (http://tcmspw.com/tcmsp.php, Version: 2.3, accessed March 2020) DrugBank and related literatures. Then the resulted targets were normalized their corresponding nomenclatures by UniPort Database (https://www.uniprot.org/, accessed March 2020).

\section{PLC Related Targets}

The differential expressed genes (DEG) of PLC patients were acquired from GEO database (https://www.ncbi.nlm.nih.gov/geo/ Series: GSE45267, Samples: GSM1100370 to GSM1100456). Genes with a $P$ value $<0.005$ and $|\log 2|>1$ were deemed as PLC related targets.

\section{Construction of Network}

To elucidate the pharmacological mechanism of HXCF in treatment of PLC, the active ingredient-target system of HXCF was generated by Cytoscape software (Version 3.7.2 Boston, MA, USA). Plugin Bisogenet of Cytoscape was used to build Protein-Protein Interaction (PPI) from Human Protein Reference Database (HPRD), Database of Interacting Proteins (DIP ${ }^{\mathrm{TM}}$ ), Biological General Repository for Interaction Datasets (BioGRID), biomolecular interaction network database (BIND), IntAct Molecular Interaction Database (IntAct) and Molecular INTeraction database (MINT). All network was visualized by Cytoscape software (Version 3.7.2 Boston, MA, USA).

\section{Network Merge}

The PPI network of HXCF was merged by Cytoscape software. And the nodes with topological importance in the interaction network were screened by calculating Betweenness Centrality (BC), Degree Centrality (DC), Eigenvector Centrality (EC) and Closeness Centrality (CC), which represent the topological 
significance. What's more, the parameters also have been reported about their definitions and computational formulas and used in network pharmacology and systems pharmacology. Local average connectivity-based method (LAC), and Network Centrality (NC) with the Cytoscape plugin CytoNCA.

\section{Bioinformatic Analysis}

The Database for Annotation, Visualization and Integrated Discovery (DAVID https://david.ncifcrf.gov/ version 6.8)[19] is a website providing a comprehensive set of functional annotation tools to understand biological meaning behind large list of genes and carrying out GO enrichment analysis and Kyoto Encyclopedia of Genes and Genomes (KEGG) enrichment analysis.In this study, top 10 of GO analysis in molecular function (MF), biological process (BP) and cellular component (CC) as well as KEGG were listed. Eventually, we used GOplot package[20] and cytoscape for visualization.

\section{Molecule Docking}

The most maximum betweenness centrality molecule and corresponding ligands were chose to dock. The crystal structure of active molecules were draw by Protein Data Bank[21,22] (PDB: 2gmx[23], 1p9m[24]). Through Chemical Book (https://www.rcsb.org/ accessed May 2020) database, the small molecules were saved as mol files uniformly. The spatial structure convert it to pdb format after checked in the PyMol software (https://pymol.org/version version 2.3). For increasing accuracy, Auto Tools, as well as Autodock Vina (http://vina.scripps.edu/index.html version 1.2), was employed to conduct docking. After pretreatment of the excess protein chain and ligands deletion, the water molecules removement as well as Gasteiger charge calculation from molecule ligand complex, the Autodock Vina was employed to dock between small molecules with proteins. The dominant conformation was analyzed and plotted with Schrodinger software (https://www.schrodinger.com accessed May 2020).

\section{Results}

1. Demographic characteristics of patients with PLC.

In this study, the information of 524 patients with PLC were collected from $1^{\text {st }}$ January 2011 to $30^{\text {th }}$ July 2019 at Affiliated Hospital of Hunan Academy of Traditional Chinese Medicine. Eventually, there were 332 individuals meeting our research criteria. what's more, 140 patients with no more than cumulative 6 months $\mathrm{CM}$ treatment were regarded as less-CM group. The rest who received $\mathrm{CM}$ treatment more than cumulative 6 months were defined as CM group. The patients' treatment method, percutaneous ablation, differed between these two groups ( $p$-value $<0.05$, Table 1 ). To decrease potential bias, propensity score matching (PSM) method was used to match individuals based on age, gender, surgery, transcatheter arterial chemoembolization, targeted therapy, radiotherapy, percutaneous ablation and chemotherapy. As presented in table 1, there were no significant differences between the matched two groups.

2. Univariate and multivariate analysis. 
Univariate and multivariate Cox proportional hazard model was used to estimate the risk of overall mortality among liver cancer patients. As shown in the table 2, serum AFP $(>200 \mu g / L)(P<0.05)$ were significantly related to the reduced median overall survival. In contrast, taking CM more than 6 months were protective factors.

\section{Survival Analysis}

As shown in fig 2, the median survival time of CM and Less-CM groups were 26.6 months vs. 16.0 months. In addition, the 1-, 2-, and 5- overall survival rate was $79.23 \% 33.85 \% 13.08 \%$ vs. $56.92 \% 4.62 \%$, $2.31 \%$. The Long-rank illustrated that it's significantly different between two groups in terms of OS.

\section{Identification of core drug}

In total, 1034 of prescriptions from $130 \mathrm{CM}$ group were conducted, which is consisted of 293 kinds of drugs. Based on the patients' syndrome differentiation, the prescriptions were changed every 5 to 30 days. And every prescription had 10 to 30 kinds of drugs. Then TCMIPA was utilized to carry out further analysis. licorice (GC), Hedysarum Multijugum Maxim. (HQ), Poria Cocos (Schw.) Wolf. (FL), Radix Bupleuri (CH), Hedyotis Diffusae Herba (BHSSC), Scutellariae Barbatae Herba (BZL), Artemisiae Scopariae Herba (YC), Polygoni Cuspidati Rhizoma Et Radix (HZ), Fructus Ligustri Lucidi (NZZ) were identified as HXCF.

\section{Screening of active ingredients}

By exploring the database, we identified that the HXCF contains 1125 chemicals totally. Based on the three significant parameter, $\mathrm{OB}, \mathrm{DL}$ and $\mathrm{HL}, 189$ ingredients satisfying with $\mathrm{OB} \geq 30 \% \triangle \mathrm{DL} \geq 0.18$ and $\mathrm{DL} \geq 4$ were filtered out, including 7 species of BHSSC 29 species of BZL 14 species of $\mathrm{CH} 14$ species of FL 76 species of GC 16 species of HQ 9 species of HZ 11 species of NZZ 13 species of YC. After deleting the duplicates, a total of 153 ingredients were filtered out, which can be seen in Fig 3. As presented in table 3 , the detail information of parts of candidate ingredients were presented as examples.

\section{Targets Identification}

Predicted by TCMSP database, 243 targets were identified as candidate targets of HXCF. And 1055 DEG were identified as the related targets of PLC from GEO database. As shown in fig 4, volcano plot and heatmap were created to exhibit the contribution of DEG.

\section{Compound-Target Network Analysis}

As presented in fig 5, 77 putative targets and 116 bioactive ingredients from HXCF were employed to construct a compound-target interaction network (C-T network). As shown in figure, the C-T network consists of 193 nodes and 529 edges. The result elucidates the compound-targets interaction.

8. Protein-Protein Interaction Analysis 
In order to explore the potential mechanisms of HXCF in treating PLC, Protein-Protein Interaction (PPI) was constructed. As presented in fig 6, the PPI network consists of 2148 nodes and 47330 edges. The media degree of nodes is 24 . In addition, the nodes with more than 74 degrees were identified as significant targets. The median values of DC, BC, CC, EC, LAC, and NC were 24, 560.60, 0.4179, 0.0086,

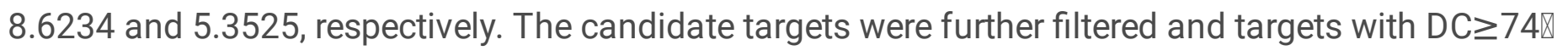
$\mathrm{BC} \geq 500$ were identified. In total, 55 genes were eventually identified for HXCF against PLC, such as VCAM1, MYC and so on.GO and KEGG enrichment analysis

To further elaborate the mechanism of HXCF to treat PLC, GO and KEGG enrichment analysis were carried out on the corresponding targets. In total, 1036 terms of GO were significantly enriched with FDR<0.05, including 27 in cellular component, 946 in biological process and 63 molecular function. The highly enriched GO terms included responding to response to oxidative stress, cyclin-dependent protein kinase holoenzyme complex and protein kinase regulator activity. The top $10 \mathrm{GO}$ entries in BC, CC, and MF and related genes were and related genes were presented in fig 7.

To investigate the pathway-level mechanisms of HXCF, The KEGG enrichment analysis was performed. 75 significantly enriched pathways (FDR $<0.05$ ) were identified. Top 10 were listed, including fluid shear stress, IL-17 signaling pathway, AGE-RAGE signaling pathway, cellular senescence, TNF signaling pathway, p53 signaling pathway, cell cycle, steroid hormone biosynthesis, Th17 cell differentiation and metabolism of xenobiotics by cytochrome.

\section{Molecular docking}

Two targets, JUN and IL-6 and corresponding small molecules were stimulated by molecular docking. The result of docking was analyzed by Pymol software. The binding affinity of small molecular were presented in table 4 . The result showed that hydrogen bonding was the main form of interaction. As shown in fig 8, we can see the interaction mode with key amino acids and their binding at the active site.

\section{Discussion}

Owing to the advanced stages of diagnosis, patients with PLC always have a poor prognosis[25,26]. Currently, the main therapies for PLC patients are surgery, TACE, radiotherapy, targeted therapy and immune therapy. Whereas, the effect is limited. The 1-year survival rate of PLC is only $38 \%[27]$. CM has been employed to treat disease for a long time in Southeast Asia[28-31]. As for the aspects of limiting symptoms, reducing treatment associated side effects, inhibiting tumor growth and altering key intracellular signaling pathways, many studies have been proved that $\mathrm{CM}$ has advantages for patients with PLC[32-34].In this retrospective study, the overall survival of patients receiving CM therapy more than 6 months was prolonged. In addition, the serum of AFP is also an independent factor affecting the survival time of patients with PLC.

Reactive oxygen species (ROS) is increasingly identified as vital signaling molecules implicated in a number of biological process which are significant for cellular homeostasis. Several studies have shown 
that ROS can regulate activities of several proteins and signal pathways, e.g. vascular endothelial growth factor (VEGF), HIF-1a, PI3K/Akt and MAPK pathways, which is responsible for liver tumor cell differentiation, angiogenesis and so on[35-38]. Serine/threonine protein kinases participate in DNA damage response[39]. Genome instability is one of the hallmarks of cancer[40]. Therefore, we suspect that serine/threonine protein kinase is one of the preferred targets for the treatment of PLC.

Pathways analysis suggest that HXCF regulates the tumor microenvironment and tumor itself to against PLC. Fluid shear stress is one of the mechanical forces caused by blood flowing in the body to the blood vessel『which is not restricted only to the pathology of atherosclerosis, but also amount of cancers. Cancer cells manage a range of physical and chemical stimulation in the tumor microenvironment, including fluid shear stress[41]. Likewise, shear stress imposed by tumor microenvironment regulates the behavior of a variety of host and cancer cells[42]. These physical forces are able to depress blood and lymphatic vessels, reducing perfusion rates and creating hypoxia[43]. When imposed directly on cancer cells, they raise the ability of cells' invasive and metastatic potential[44,45]. Experimental evidence have reported that inhibiting these physical forces can increase therapeutic outcomes in many cancers[46-49]. However, there are few reports on the effect of fluid shear stress on liver cancer. This may be the potential mechanism for the treatment of liver cancer.

Incidence of PLC is closely related to choric virus infection and/or cirrhosis, which present tumor cells existing in an inflammatory microenvironment[50-52]. Interleukin-17 (IL-17) family produced by CD4+ T helper (Th) cells plays a vital role in orchestrating host immune responses[53]. Th17 cells have powerful pro-inflammatory properties and play an active role in liver cancer[54]. Emerging evidence indicates that IL-17 family members play an important role in coordinating local tissue inflammation largely through the induced release of neutrophil-mobilizing cytokines and proinflammatory[53].

Receptor for advanced glycation end products (RAGE) interacts with its ligands to stimulate the activation of a variety of signaling pathways such as PI3K,/AKT/mTOR, NF-KB, and so on[55-57]. RAGE is highly expressed in liver cancer[58]. Accumulating data indicate that RAGE participate in autophagy and apotosis[59,60]. Recent studies have shown that tumor necrosis factor (TNF) is the common and representative inflammatory cytokines in tumor microenvironment, which involved in growth differentiation invasion and metastasis in the multistep processes of tumorigenesis[61-63]. Many studies reported that TNF families are closely related to the prognosis of PLC[64,65]. P53, mutated in PLC, is a pivotal tumor suppressor gene and a significant mainstay in our body's natural anti-cancer defense[66]. Several studies have shown that P53 in liver cancer can function to restrain tumor development[67,68].

Molecular docking results predict that amount of ingredients in HXCF exerts therapeutic effects against PLC, at least in part, by modulating the function of c-JUN and IL-6.

\section{Conclusion}

In this study, we concluded that CM taken more than 6 months benefits for the patients with PLC. What's more, data mining and system pharmacology was applied to unveil biochemistry basis and potential 
mechanisms of CM that really benefit foe the penefit for the patient with PLC. In total 153 ingredients from 9 core drugs were screened out. The mechanism of $\mathrm{HXCH}$ in treating PLC is involved in several targets including VCAM1, MYC as well as several biological pathways, such as fluid shear stress, IL-17 signaling pathway, AGE-RAGE signaling pathway, cellular senescence, TNF signaling pathway, p53 signaling pathway. However, it's worthy to note that further experimental verifications of above predicted results are required for their clinical translational potential in the future.

\section{Declarations}

\section{Acknowledgements}

We thank all of participating researchers and our study staff.

\section{Authors' contributions}

$\mathrm{ZZ}$ and PHZ contributed to the conception of this manuscript and wrote the draft; XFT contributed to this manuscript revision, the data collection and funding; $\mathrm{YW}$ and $\mathrm{WHZ}$ contributed to the data collection, ST contributed to the manuscript revision. WHG and ZQ helped with revision; All authors read and approved the final manuscript.

\section{Funding}

This work was supported by the National Natural Science Foundation of China $(8160360,81473617)$, Natural Science Foundation of Hunan Province (2016JJ6113); Outstanding Youth Project of Hunan Provincial Department of Education (18B247); Innovation and Entrepreneurship Training Program for College Students in Hunan Province (1684). The funding bodies had no involvement in the concept, design, analysis or writing the manuscript.

\section{Availability of data and materials}

The datasets generated and/or analyzed during the study are not publicly available to protect subject confidentiality but are available from the corresponding author on reasonable request.

\section{Ethics approval and consent to participate}

All procedures performed in studies involving human participants were in accordance with the ethical standards of the Air Force Medical University Ethics Committee and the 1964 Helsinki declaration and its later amendments or comparable ethical standards. The study protocol and informed consent form were reviewed and approved by the Affiliated Hospital of Hunan Academy of Traditional Chinese Medicine [IRB No.: (202004)26]. Written informed consent was provided to all subjects prior to the start of the study.

\section{Consent for publication}

Not applicable. 


\section{Competing Interests}

The authors declare no conflict of interest, financial or otherwise.

\section{References}

1. Ahmed S, de Souza NN, Qiao W, Kasai M, Keem LJ, Shelat VG: Quality of Life in Hepatocellular Carcinoma Patients Treated with Transarterial Chemoembolization. HPB Surg, 2016; 2016: 6120143

2. Bray F, Ferlay J, Soerjomataram I, Siegel RL, Torre LA, Jemal A: Global cancer statistics 2018: GLOBOCAN estimates of incidence and mortality worldwide for 36 cancers in 185 countries. CA Cancer J Clin, 2018; 68: 394-424

3. Lavanchy D: Worldwide epidemiology of HBV infection, disease burden, and vaccine prevention. J Clin Virol, 2005; 34 Suppl 1: S1-3

4. Cheung C, Law MF, Chao DC et al: Prevention of hepatitis B virus reactivation in patients with hematological malignancies and resolved hepatitis B virus infection: a systematic review and metaanalysis. J Dig Dis, 2020;

5. Jemal A, Murray T, Ward E et al: Cancer statistics, 2005. CA Cancer J Clin, 2005; 55: 10-30

6. Jung KW, Won YJ, Park S et al: Cancer statistics in Korea: incidence, mortality and survival in 2005. J Korean Med Sci, 2009; 24: 995-1003

7. Sun JH, Sun F, Yan B, Li JY, Xin L: Data mining and systematic pharmacology to reveal the mechanisms of traditional Chinese medicine in Mycoplasma pneumoniae pneumonia treatment. Biomed Pharmacother, 2020; 125: 109900

8. Dai QL, Jiang F, Wang J, Gong CL, Shi MY: [Based on traditional Chinese medicine inheritance support system to analyze the regularity of umbilicus application to treat ascites due to cirrhosis]. Zhongguo Zhong Yao Za Zhi, 2018; 43: 4541-6

9. Sun ZX, Zhang PP, Gao WL, Dai GH: [Analysis on medication rules of modern traditional Chinese medicines in treating palpitations based on traditional Chinese medicine inheritance support system]. Zhongguo Zhong Yao Za Zhi, 2017; 42: 385-9

10. He LJ, Zhu XD: [Analysis on regularity of prescriptions in "a guide to clinical practice with medical record" for diarrhoea based on traditional Chinese medicine inheritance support system]. Zhongguo Zhong Yao Za Zhi, 2016; 41: 2344-9

11. Patra BG, Maroufy V, Soltanalizadeh B et al: A content-based literature recommendation system for datasets to improve data reusability - A case study on Gene Expression Omnibus (GEO) datasets. J Biomed Inform, 2020; 104: 103399

12. Li $X, X u X$, Wang $J$ et al: A system-level investigation into the mechanisms of Chinese Traditional Medicine: Compound Danshen Formula for cardiovascular disease treatment. PLoS One, 2012; 7: e43918 
13. Zhu N, Hou J: Exploring the mechanism of action Xianlingubao Prescription in the treatment of osteoporosis by network pharmacology. Comput Biol Chem, 2020; 85: 107240

14. Zhou W, Wu J, Zhu Y et al: Study on the mechanisms of compound Kushen injection for the treatment of gastric cancer based on network pharmacology. BMC Complement Med Ther, 2020; 20: 6

15. Liu H, Wang J, Zhou W, Wang Y, Yang L: Systems approaches and polypharmacology for drug discovery from herbal medicines: an example using licorice. J Ethnopharmacol, 2013; 146: 773-93

16. Tian S, Wang J, Li Y, Li D, Xu L, Hou T: The application of in silico drug-likeness predictions in pharmaceutical research. Adv Drug Deliv Rev, 2015; 86: 2-10

17. Wishart DS, Knox C, Guo AC et al: DrugBank: a knowledgebase for drugs, drug actions and drug targets. Nucleic Acids Res, 2008; 36: D901-6

18. Yan G, Wang X, Chen Z et al: In-silico ADME Studies for New Drug Discovery: From Chemical Compounds to Chinese Herbal Medicines. Curr Drug Metab, 2017; 18: 535-9

19. Huang da W, Sherman BT, Lempicki RA: Systematic and integrative analysis of large gene lists using DAVID bioinformatics resources. Nat Protoc, 2009; 4: 44-57

20. Walter W, Sánchez-Cabo F, Ricote M: GOplot: an R package for visually combining expression data with functional analysis. Bioinformatics, 2015; 31: 2912-4

21. Berman HM, Westbrook J, Feng Z et al: The Protein Data Bank. Nucleic Acids Res, 2000; 28: 235-42

22. Noguchi T, Akiyama Y: PDB-REPRDB: a database of representative protein chains from the Protein Data Bank (PDB) in 2003. Nucleic Acids Res, 2003; 31: 492-3

23. Szczepankiewicz BG, Kosogof C, Nelson LT et al: Aminopyridine-based c-Jun N-terminal kinase inhibitors with cellular activity and minimal cross-kinase activity. J Med Chem, 2006; 49: 3563-80

24. Boulanger MJ, Chow DC, Brevnova EE, Garcia KC: Hexameric structure and assembly of the interleukin-6/IL-6 alpha-receptor/gp130 complex. Science, 2003; 300: 2101-4

25. Shiani A, Narayanan S, Pena L, Friedman M: The Role of Diagnosis and Treatment of Underlying Liver Disease for the Prognosis of Primary Liver Cancer. Cancer Control, 2017; 24: 1073274817729240

26. Bruix J, Reig M, Sherman M: Evidence-Based Diagnosis, Staging, and Treatment of Patients With Hepatocellular Carcinoma. Gastroenterology, 2016; 150: 835-53

27. Momin BR, Pinheiro PS, Carreira H, Li C, Weir HK: Liver cancer survival in the United States by race and stage (2001-2009): Findings from the CONCORD-2 study. Cancer, 2017; 123 Suppl 24: 5059-78

28. Wu X, Gao S, Guo Y: Attitude of Students in Shanghai University of Traditional Chinese Medicine Toward Donation After Cardiac Death. Transplant Proc, 2020;

29. Ren JL, Zhang AH, Wang XJ: Traditional Chinese Medicine for COVID-19 Treatment. Pharmacol Res, $2020: 104743$

30. Wang M, Ye Q, Mao D, Li H: Research Progress in Liver-Regenerating Microenvironment and DNA Methylation in Hepatocellular Carcinoma: The Role of Traditional Chinese Medicine. Med Sci Monit, 
2020; 26: e920310

31. Ren JL, Zhang AH, Wang XJ: Traditional Chinese medicine for COVID-19 treatment. Pharmacol Res, 2020; 155: 104743

32. Liu X, Li M, Wang X et al: Effects of adjuvant traditional Chinese medicine therapy on long-term survival in patients with hepatocellular carcinoma. Phytomedicine, 2019; 62: 152930

33. Xi SY, Minuk GY: Role of traditional Chinese medicine in the management of patients with hepatocellular carcinoma. World J Hepatol, 2018; 10: 799-806

34. Xie HP, Liu ZP, Zhang JS et al: Traditional Chinese Medicine Syndrome Patterns and Their Association with Hepatitis B Surface Antigen Levels during the Natural History of Chronic Hepatitis B Virus Infection. Evid Based Complement Alternat Med, 2018; 2018: 7482593

35. Zhao J, Zhang L, Li J et al: A novel pyrazolone-based derivative induces apoptosis in human esophageal cells via reactive oxygen species (ROS) generation and caspase-dependent mitochondria-mediated pathway. Chem Biol Interact, 2015; 231: 1-9

36. Menzies FM, Fleming A, Rubinsztein DC: Compromised autophagy and neurodegenerative diseases. Nat Rev Neurosci, 2015; 16: 345-57

37. Muthukkaruppan VR, Kubai L, Auerbach R: Tumor-induced neovascularization in the mouse eye. J Natl Cancer Inst, 1982; 69: 699-708

38. Potente M, Gerhardt H, Carmeliet P: Basic and therapeutic aspects of angiogenesis. Cell, 2011; 146: 873-87

39. Olsen JV, Blagoev B, Gnad F et al: Global, in vivo, and site-specific phosphorylation dynamics in signaling networks. Cell, 2006; 127: 635-48

40. Hanahan D, Weinberg RA: Hallmarks of cancer: the next generation. Cell, 2011; 144: 646-74

41. Jain RK: An indirect way to tame cancer. Sci Am, 2014; 310: 46-53

42. Jain RK: Normalizing tumor microenvironment to treat cancer: bench to bedside to biomarkers. J Clin Oncol, 2013; 31: 2205-18

43. Jain RK, Martin JD, Stylianopoulos T: The role of mechanical forces in tumor growth and therapy. Annu Rev Biomed Eng, 2014; 16: 321-46

44. Stylianopoulos T, Martin JD, Snuderl M, Mpekris F, Jain SR, Jain RK: Coevolution of solid stress and interstitial fluid pressure in tumors during progression: implications for vascular collapse. Cancer Res, 2013; 73: 3833-41

45. Helmlinger G, Netti PA, Lichtenbeld HC, Melder RJ, Jain RK: Solid stress inhibits the growth of multicellular tumor spheroids. Nat Biotechnol, 1997; 15: 778-83

46. Novak CM, Horst EN, Taylor CC, Liu CZ, Mehta G: Fluid shear stress stimulates breast cancer cells to display invasive and chemoresistant phenotypes while upregulating PLAU in a 3D bioreactor. Biotechnol Bioeng, 2019; 116: 3084-97

47. Hyler AR, Baudoin NC, Brown MS et al: Fluid shear stress impacts ovarian cancer cell viability, subcellular organization, and promotes genomic instability. PLoS One, 2018; 13: e0194170 
48. Zhang W, Mao S, He Z, Wu Z, Lin JM: In Situ Monitoring of Fluid Shear Stress Enhanced Adherence of Bacteria to Cancer Cells on Microfluidic Chip. Anal Chem, 2019; 91: 5973-9

49. Triantafillu UL, Park S, Klaassen NL, Raddatz AD, Kim Y: Fluid shear stress induces cancer stem celllike phenotype in MCF7 breast cancer cell line without inducing epithelial to mesenchymal transition. Int J Oncol, 2017; 50: 993-1001

50. Lanser L, Kink P, Egger EM et al: Inflammation-Induced Tryptophan Breakdown is Related With Anemia, Fatigue, and Depression in Cancer. Front Immunol, 2020; 11: 249

51. Lichtenstern CR, Ngu RK, Shalapour S, Karin M: Immunotherapy, Inflammation and Colorectal Cancer. Cells, 2020; 9

52. Coussens LM, Werb Z: Inflammation and cancer. Nature, 2002; 420: 860-7

53. Kolls JK, Lindén A: Interleukin-17 family members and inflammation. Immunity, 2004; 21: 467-76

54. Zhang JP, Yan J, Xu J et al: Increased intratumoral IL-17-producing cells correlate with poor survival in hepatocellular carcinoma patients. J Hepatol, 2009; 50: 980-9

55. Yan SF, Ramasamy R, Schmidt AM: Receptor for AGE (RAGE) and its ligands-cast into leading roles in diabetes and the inflammatory response. J Mol Med (Berl), 2009; 87: 235-47

56. Palanissami G, Paul S: RAGE and Its Ligands: Molecular Interplay Between Glycation, Inflammation, and Hallmarks of Cancer-a Review. Horm Cancer, 2018; 9: 295-325

57. Tesarova P, Cabinakova M, Mikulova V, Zima T, Kalousova M: RAGE and its ligands in cancer culprits, biomarkers, or therapeutic targets. Neoplasma, 2015; 62: 353-64

58. Kohles N, Nagel D, Jüngst D, Stieber P, Holdenrieder S: Predictive value of immunogenic cell death biomarkers HMGB1, sRAGE, and DNase in liver cancer patients receiving transarterial chemoembolization therapy. Tumour Biol, 2012; 33: 2401-9

59. Kang R, Tang D, Schapiro NE et al: The receptor for advanced glycation end products (RAGE) sustains autophagy and limits apoptosis, promoting pancreatic tumor cell survival. Cell Death Differ, 2010; 17: 666-76

60. Li J, Wu PW, Zhou Y et al: Rage induces hepatocellular carcinoma proliferation and sorafenib resistance by modulating autophagy. Cell Death Dis, 2018; 9: 225

61. Balkwill F: Tumor necrosis factor or tumor promoting factor. Cytokine Growth Factor Rev, 2002; 13: $135-41$

62. Shi HZ, Ren P, Lu QJ, Niedrgethmnn M, Wu GY: Association between EGF, TGF- $\beta 1$ and TNF-a gene polymorphisms and hepatocellular carcinoma. Asian Pac J Cancer Prev, 2012; 13: 6217-20

63. Dogra G, Chakravarti A, Kar P, Chawla YK: Polymorphism of tumor necrosis factor-a and interleukin10 gene promoter region in chronic hepatitis $C$ virus patients and their effect on pegylated interferona therapy response. Hum Immunol, 2011; 72: 935-9

64. Wang JJ, Wang ZB, Tan TC: Association of CTLA-4, TNF alpha and IL 10 polymorphisms with susceptibility to hepatocellular carcinoma. Scand J Immunol, 2019; 90: e12819 
65. Zhang M, Hu J, Li H et al: High TNF-a and/or p38MAPK expression predicts a favourable prognosis in patients with T1NOMO hepatocellular carcinoma: An immunohistochemical study. Oncol Lett, 2019; 17: 4948-56

66. Levine AJ, Oren M: The first 30 years of p53: growing ever more complex. Nat Rev Cancer, 2009; 9: 749-58

67. Blagih J, Buck MD, Vousden KH: p53, cancer and the immune response. J Cell Sci, 2020; 133

68. Chen Y, Chen G, Li J et al: Association of Tumor Protein p53 and Ataxia-Telangiectasia Mutated Comutation With Response to Immune Checkpoint Inhibitors and Mortality in Patients With NonSmall Cell Lung Cancer. JAMA Netw Open, 2019; 2: e1911895

\section{Tables}

Table 1 Baseline characteristics between two groups 


\begin{tabular}{|c|c|c|c|c|c|c|}
\hline \multirow[t]{2}{*}{ Characteristics } & \multicolumn{2}{|c|}{ Total subjects } & \multirow{2}{*}{$\begin{array}{l}\mathrm{P}- \\
\text { value }\end{array}$} & \multicolumn{2}{|c|}{ Matched subjects } & \multirow{2}{*}{$\begin{array}{l}\text { P- } \\
\text { value }\end{array}$} \\
\hline & $\mathrm{CM}$ & Less-CM & & $\mathrm{CM}$ & Less-CM & \\
\hline Age(Mean $\pm S D)$ & $55.44 \pm 11.92$ & $56.53 \pm 12.13$ & 0.417 & $55.46 \pm 12.077$ & $56.81 \pm 12.06$ & 0.539 \\
\hline \multicolumn{7}{|l|}{ Gender } \\
\hline Male & 154 & 116 & \multirow[t]{2}{*}{0.541} & 106 & 107 & \multirow[t]{2}{*}{0.872} \\
\hline Female & 38 & 24 & & 24 & 23 & \\
\hline \multicolumn{7}{|l|}{$\mathrm{HBV}$ or HCV } \\
\hline Yes & 166 & 119 & \multirow[t]{2}{*}{0.704} & 113 & 111 & \multirow[t]{2}{*}{0.720} \\
\hline No & 26 & 21 & & 17 & 19 & \\
\hline \multicolumn{7}{|l|}{ Liver cirrhosis } \\
\hline Yes & 93 & 59 & \multirow[t]{2}{*}{0.256} & 64 & 56 & \multirow[t]{2}{*}{0.332} \\
\hline No & 99 & 81 & & 66 & 64 & \\
\hline \multicolumn{7}{|c|}{ Child-Pugh Stage } \\
\hline A & 60 & 32 & \multirow[t]{3}{*}{0.237} & 37 & 32 & \multirow[t]{3}{*}{0.770} \\
\hline B & 104 & 84 & & 74 & 77 & \\
\hline C & 28 & 24 & & 19 & 21 & \\
\hline \multicolumn{7}{|l|}{ TNM Stage } \\
\hline$\square$ & 27 & 8 & \multirow[t]{4}{*}{0.110} & 18 & 8 & \multirow[t]{4}{*}{0.158} \\
\hline ૧ & 38 & 30 & & 21 & 29 & \\
\hline$\square$ & 83 & 68 & & 60 & 60 & \\
\hline ૧ & 44 & 34 & & 31 & 33 & \\
\hline \multicolumn{7}{|l|}{ Serum AFP } \\
\hline$\geq 200 \mathrm{ug} / \mathrm{ml}$ & 88 & 69 & \multirow[t]{2}{*}{0.534} & 64 & 67 & \multirow[t]{2}{*}{0.901} \\
\hline$₫ 200 u g / m l$ & 104 & 71 & & 66 & 63 & \\
\hline \multicolumn{7}{|l|}{ Surgery } \\
\hline Yes & 50 & 26 & \multirow[t]{2}{*}{0.435} & 29 & 25 & \multirow[t]{2}{*}{0.541} \\
\hline No & 142 & 114 & & 101 & 105 & \\
\hline \multicolumn{7}{|l|}{ TACE } \\
\hline Yes & 94 & 86 & 0.061 & 65 & 77 & 0.135 \\
\hline
\end{tabular}




\begin{tabular}{|c|c|c|c|c|c|c|}
\hline No & 97 & 54 & & 65 & 53 & \\
\hline \multicolumn{7}{|c|}{ Targeted Therapy } \\
\hline Yes & 34 & 28 & \multirow[t]{2}{*}{0.61} & 19 & 28 & \multirow[t]{2}{*}{0.147} \\
\hline No & 157 & 112 & & 111 & 102 & \\
\hline \multicolumn{7}{|c|}{$\begin{array}{l}\text { Percutaneous } \\
\text { Ablation }\end{array}$} \\
\hline Yes & 24 & 30 & \multirow[t]{2}{*}{0.029} & 22 & 22 & \multirow[t]{2}{*}{1.000} \\
\hline No & 168 & 110 & & 108 & 108 & \\
\hline \multicolumn{7}{|c|}{ Radiotherapy } \\
\hline Yes & 34 & 29 & \multirow[t]{2}{*}{0.490} & 27 & 26 & \multirow[t]{2}{*}{0.878} \\
\hline No & 158 & 111 & & 103 & 104 & \\
\hline \multicolumn{7}{|c|}{ chemotherapy } \\
\hline Yes & 16 & 14 & \multirow[t]{2}{*}{0.601} & 13 & 13 & \multirow[t]{2}{*}{1.000} \\
\hline No & 176 & 126 & & 117 & 117 & \\
\hline
\end{tabular}

$\mathrm{p}$-values were acquired by chi-square test; $\mathrm{p}$-value of age was acquired by the un-paired Student $\mathrm{t}$-test $\mathrm{A}$ significant difference $(p<0.05)$ is highlighted in bold italic.

Table 2 Univariate and Multivariate Analyses of Variables Influencing Survival of two groups

\begin{tabular}{|c|c|c|c|c|c|c|}
\hline \multirow[t]{2}{*}{ characteristics } & \multicolumn{3}{|c|}{ Univariate Analysis } & \multicolumn{3}{|c|}{ Multivariate Analysis } \\
\hline & $\begin{array}{l}\text { Hazard } \\
\text { Ratio }\end{array}$ & $95 \% \mathrm{Cl}$ & $\begin{array}{l}\mathrm{P}- \\
\text { value }\end{array}$ & $\begin{array}{l}\text { Hazard } \\
\text { Ratio }\end{array}$ & $95 \% \mathrm{Cl}$ & $\begin{array}{l}\mathrm{P} \text { - } \\
\text { value }\end{array}$ \\
\hline Age $\geq 60 /<60$ & 1.341 & $\begin{array}{l}0.943- \\
1.905\end{array}$ & 0.102 & - & - & - \\
\hline $\begin{array}{l}\text { Gender } \\
\text { female/male }\end{array}$ & 0.806 & $\begin{array}{l}0.508- \\
1.278\end{array}$ & 0.360 & - & - & - \\
\hline HCV or HBV \vs.no® & 1.141 & $\begin{array}{l}0.671- \\
1.351\end{array}$ & 0.161 & - & - & - \\
\hline $\begin{array}{l}\text { Liver cirrhosis } \\
\text { (vs.no) }\end{array}$ & 0.952 & $\begin{array}{l}0.563- \\
1.118\end{array}$ & 0.782 & - & - & - \\
\hline $\begin{array}{l}\text { Serum AFP } \\
\leq 200 />200\end{array}$ & 2.451 & $\begin{array}{l}1.699- \\
3.535\end{array}$ & 0.000 & 1.938 & $\begin{array}{l}1.350- \\
2.782\end{array}$ & 0.000 \\
\hline CM/Less-CM & 1.8884 & $\begin{array}{l}1.884- \\
2.691\end{array}$ & 0.001 & 2.507 & $\begin{array}{l}1.732- \\
3.630\end{array}$ & 0.000 \\
\hline
\end{tabular}


Table 3 Certain candidate ingredients of HXCF

\begin{tabular}{|c|c|c|c|c|c|c|}
\hline Number & Compound Name & $\mathrm{OB}$ & $\mathrm{DL}$ & $\mathrm{HL}$ & Structure & Related Drugs \\
\hline MOL000098 & quercetin & 46.43 & 0.28 & 14.4 & & All of the drugs \\
\hline MOL000358 & beta-sitosterol & 36.91 & 0.75 & 5.36 & & $\begin{array}{l}\text { BHSSC; BZL; HZ; } \\
\text { NZZ; YC }\end{array}$ \\
\hline MOL000006 & luteolin & 36.16 & 0.25 & 15.94 & & $\mathrm{BZL} ; \mathrm{HZ} ; \mathrm{NZZ}$ \\
\hline MOL000422 & kaempferol & 41.88 & 0.24 & 14.74 & & $\mathrm{CH} ; \mathrm{GC} ; \mathrm{HQ} ; \mathrm{NZZ}$ \\
\hline MOL000354 & isorhamnetin & 49.6 & 0.31 & 14.34 & & $\mathrm{CH}$; GC; HQ; YC \\
\hline MOL000449 & Stigmasterol & 43.83 & 0.76 & 5.57 & & BHSSC; BZL; CH \\
\hline MOL000239 & Jaranol & 50.83 & 0.29 & 15.5 & & $\mathrm{GC} ; \mathrm{HQ}$ \\
\hline MOL000296 & hederagenin & 36.91 & 0.75 & 5.35 & & FL; HQ \\
\hline MOL002776 & Baicalin & 40.12 & 0.75 & 17.36 & & $\mathrm{CH}$; BZL \\
\hline MOL002268 & rhein & 47.07 & 0.28 & 0.28 & & $\mathrm{HZ}$ \\
\hline MOL000279 & Cerevisterol & 37.96 & 0.77 & 5.31 & & FL \\
\hline
\end{tabular}

Table4 The result of molecular docking 


\begin{tabular}{|llll|}
\hline Chemical compound & Binding energy $(\mathrm{kcal} / \mathrm{mol})$ & hydrogen bonds & Gene \\
\hline quercetin & -8.3 & 1 & JUN \\
\hline wogonin173 & -8.3 & 2 & JUN \\
\hline luteolin & -8.6 & 2 & JUN \\
\hline beta-sitosterol & -8.2 & 1 & JUN \\
\hline Kaempferol & -8.0 & 2 & JUN \\
\hline formononetin & -8.5 & 2 & JUN \\
\hline wogonin173 & -7.1 & 3 & IL-6 \\
\hline luteolin & -8.0 & 3 & IL-6 \\
\hline quercetin & -7.9 & 1 & IL-6 \\
\hline
\end{tabular}

\section{Figures}

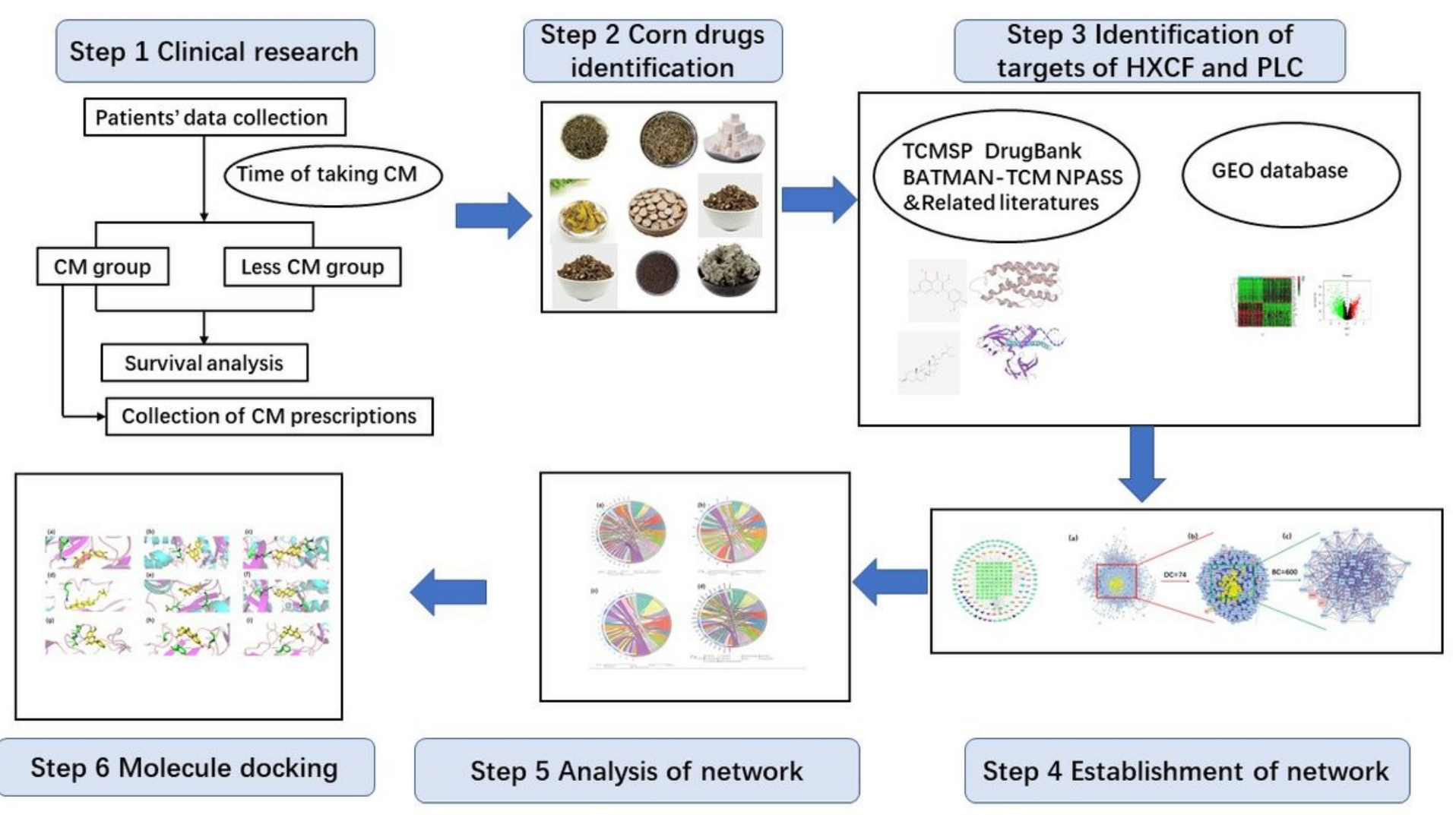

Figure 1

Workflow of clinical research and system pharmacology to explore efficiency and mechanism of CM in PLC Treatment 


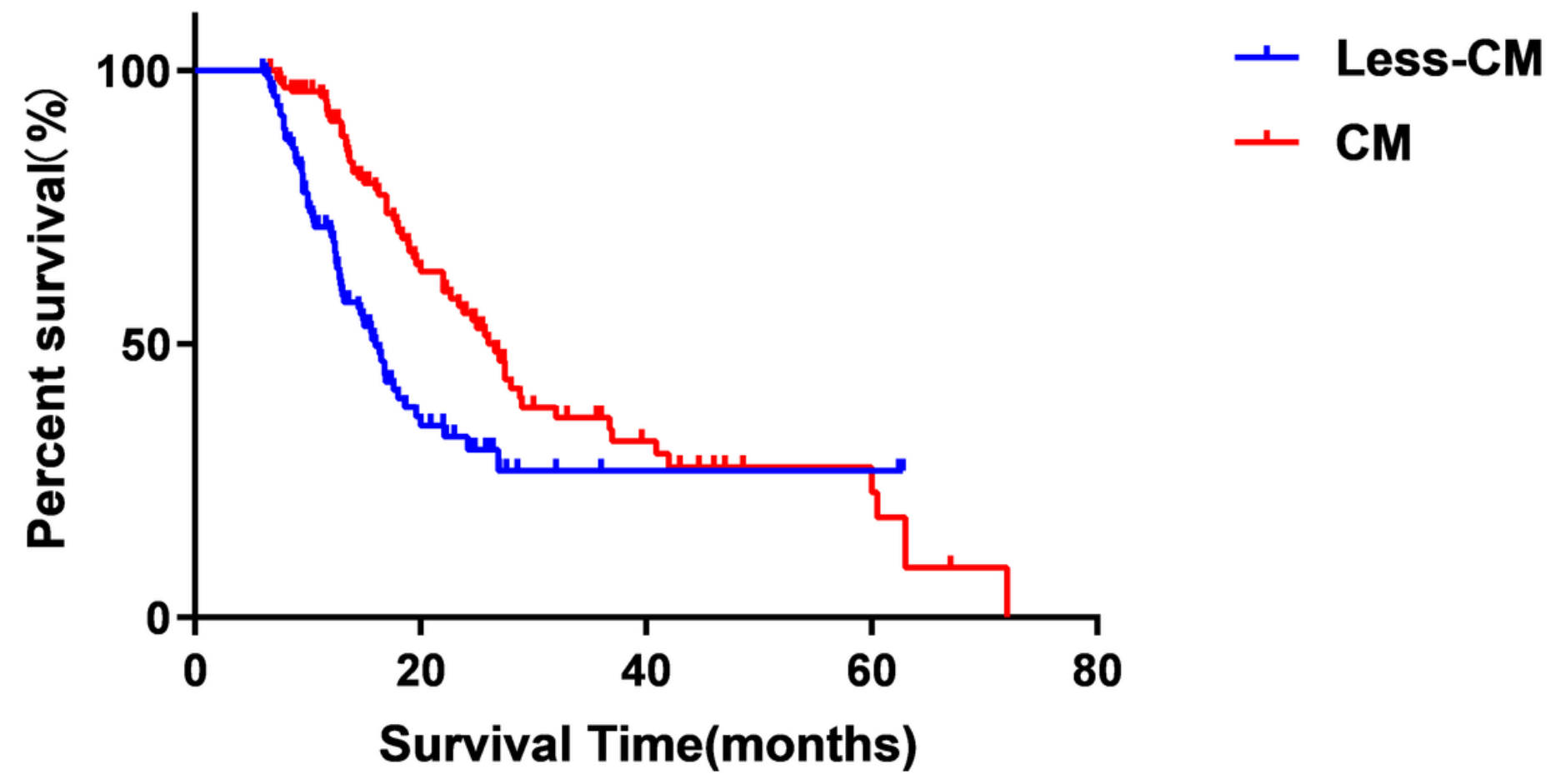

Figure 2

The survival analysis between two groups

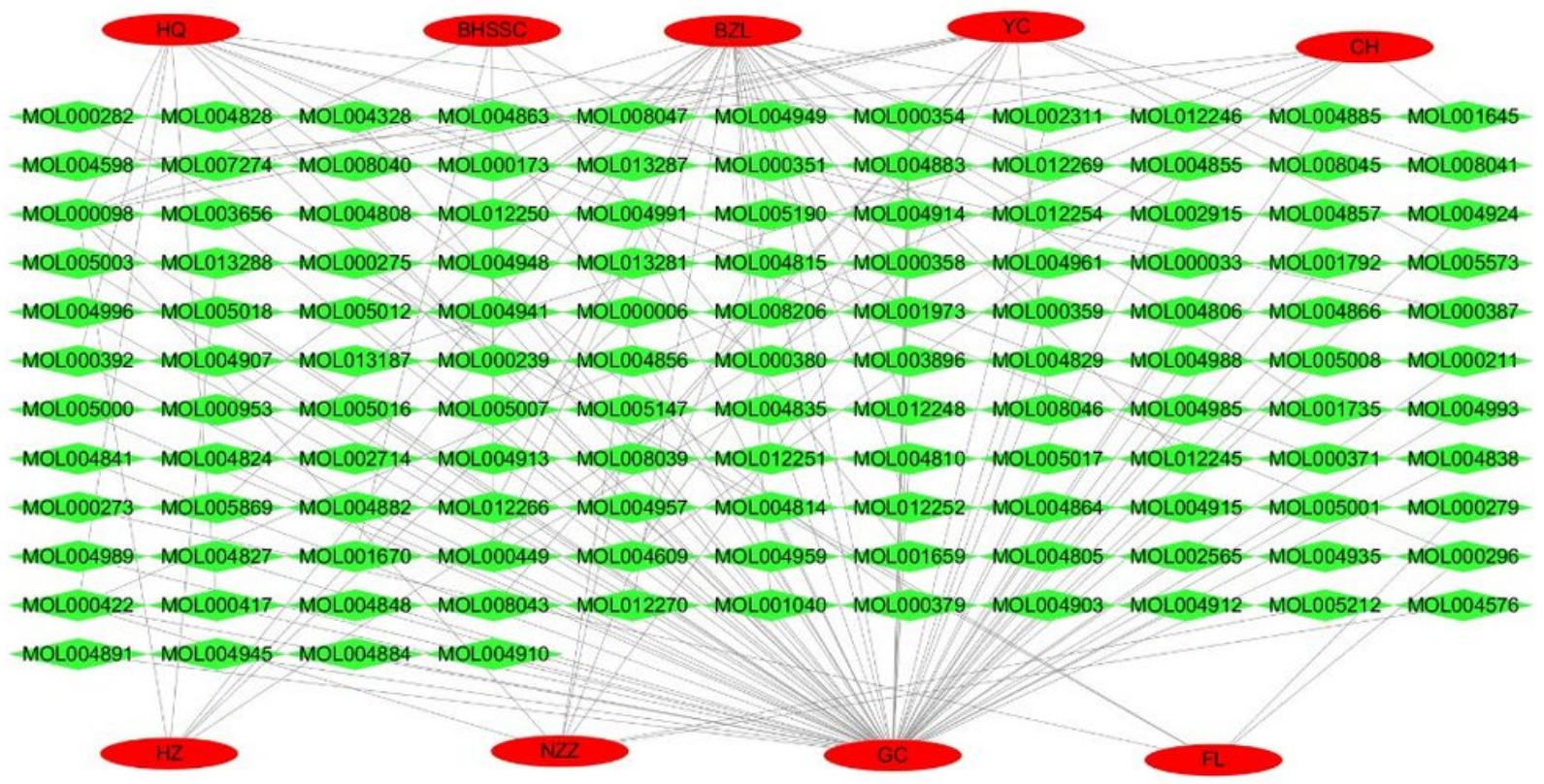

Figure 3 
Drug-ingredient network of potential bioactive constituents for HXCF. The red nodes represent drugs, and the green nodes represent ingredients of potential bioactive constituents for HXCF.

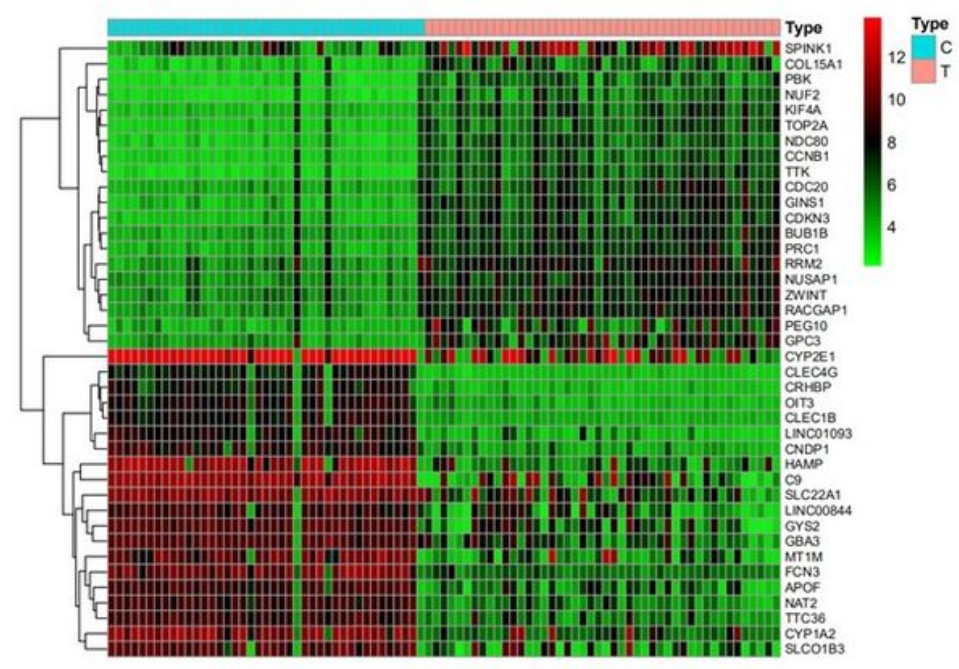

(a)

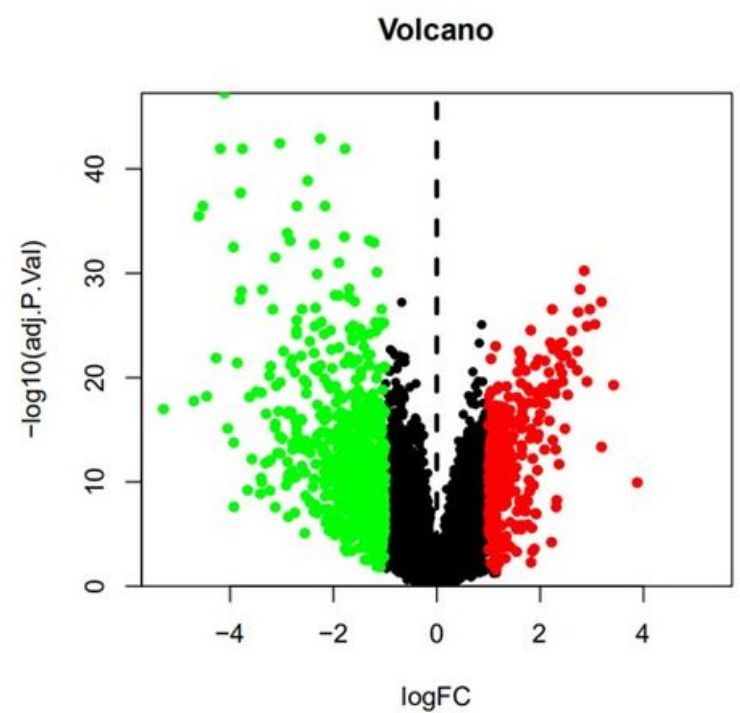

(b)

\section{Figure 4}

DEG in PLC (a)Heatmap plots showed the distribution of DEG. Pink color represented tumor group(T), while blue color represented contrasts group(C). Red color represents up-regulated genes while the green color represents downregulated genes. (b) Volcano plots showed the distribution of DEG. Upregulated genes were represented by red dots while the downregulated genes were represented by green dots, while the black dots represent indifferently expressed genes. 


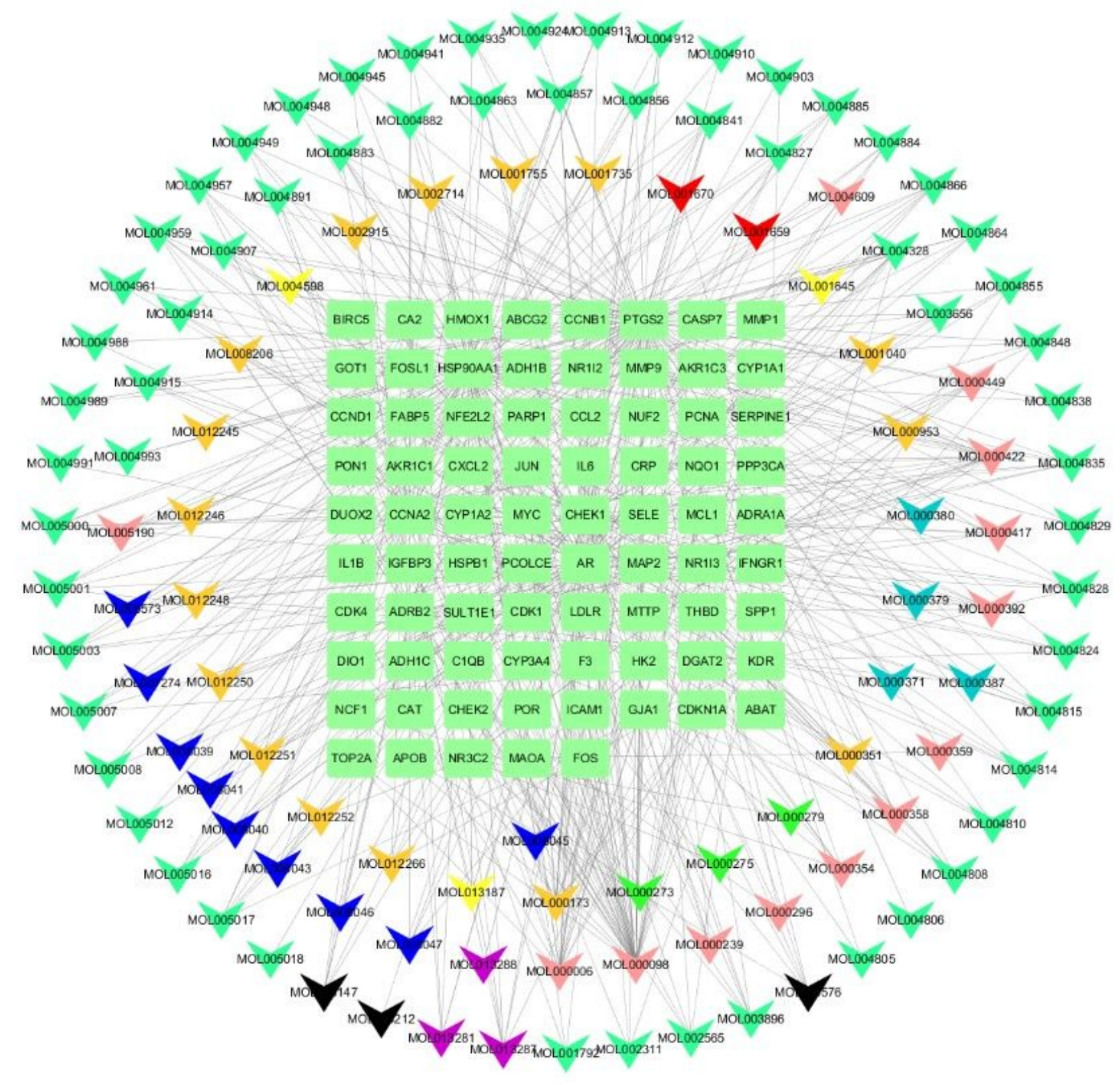

Figure 5

Compound-target network of HXCF. Squares represent targets; the red, orange, yellow, green, cyan, blue, purple, black, pink, heavy blue represents the ingredients from BHSSC, BZL, CH, FL, GC, HQ, HZ, NZZ, YC, and multiple-drug, respectively. 


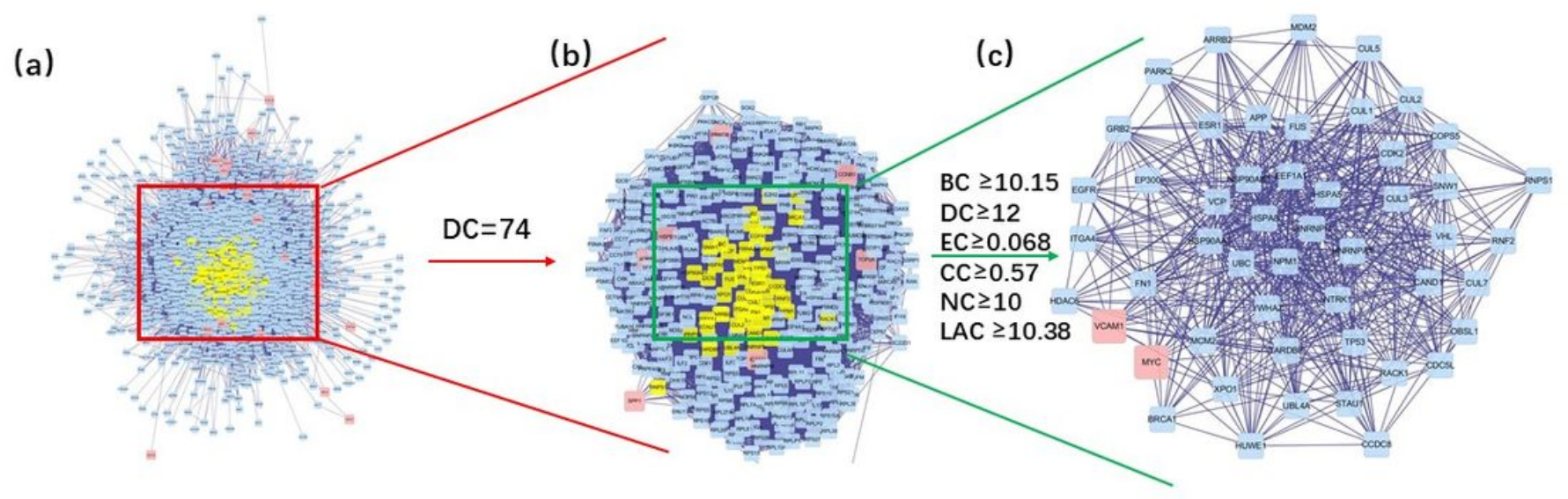

\section{Figure 6}

Identification of candidate targets of HXCF against PLC. (a) The interactive PPI network of HXCF putative targets and PLC related targets. (B) PPI network of important proteins extracted from (a). (c) PPI network of candidate HXCF targets for PLC treatment extracted from (b). The yellow dots represent selected targets, while the pink represent putative targets. 

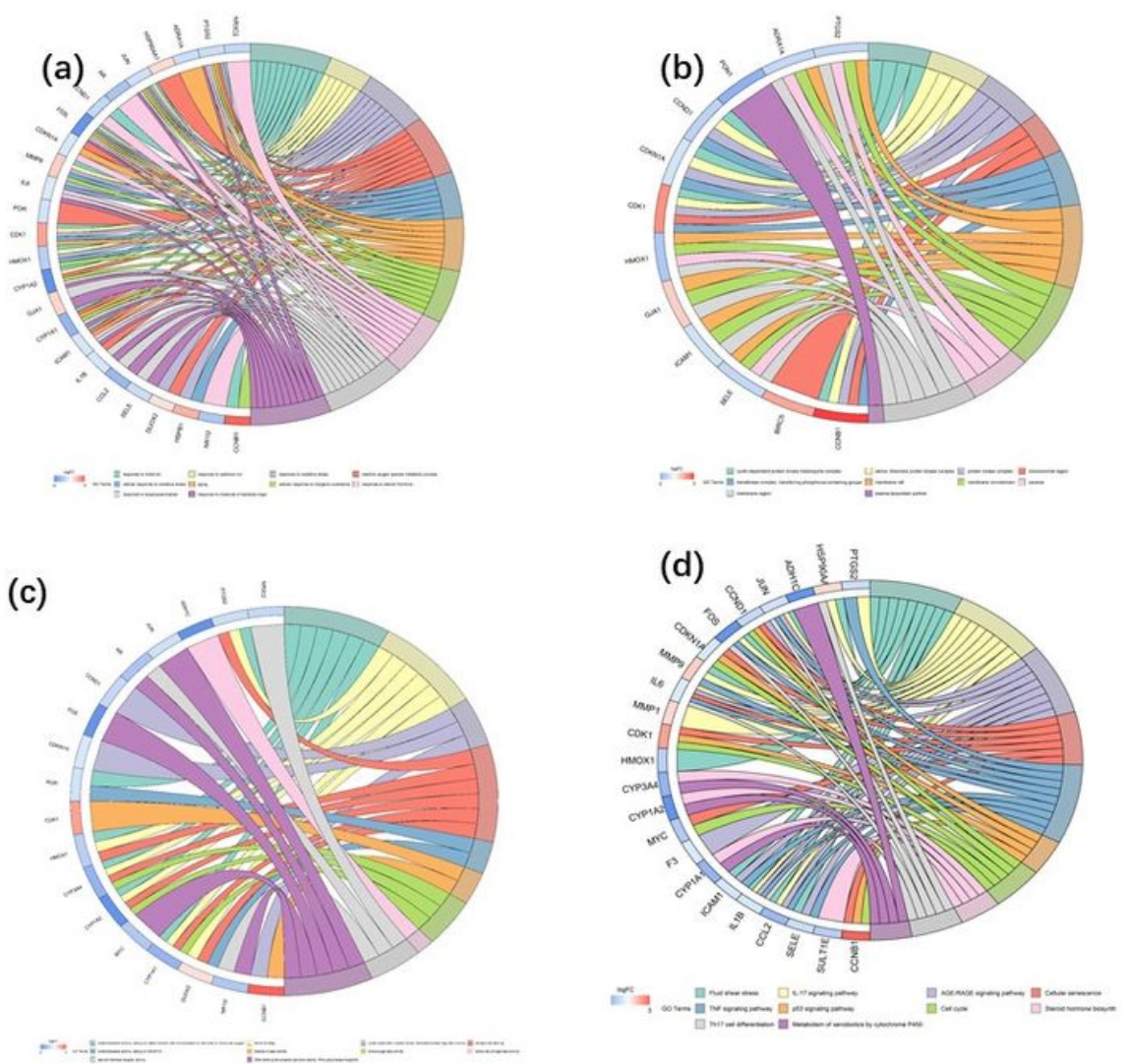

\section{Figure 7}

Circular visualization of the enriched GO and KEGG terms. (a) represents terms in BC. (b) represents terms in CC. (c) represents terms in MF. (d) represents terms in KEGG.

(a)

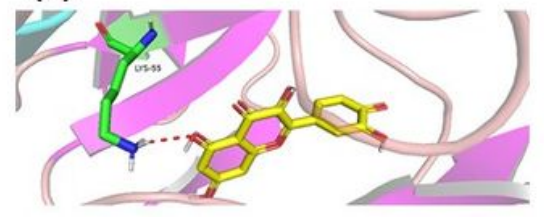

(d)

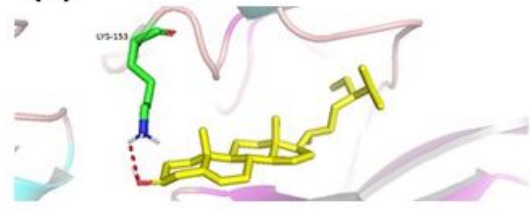

(g)

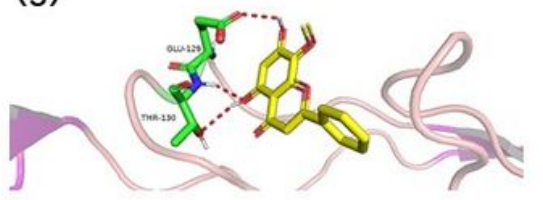

(b)

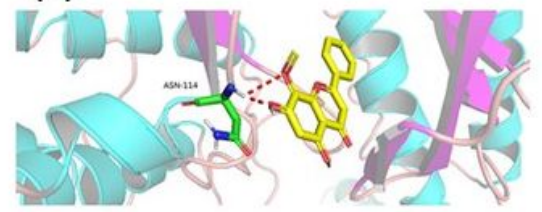

(e)

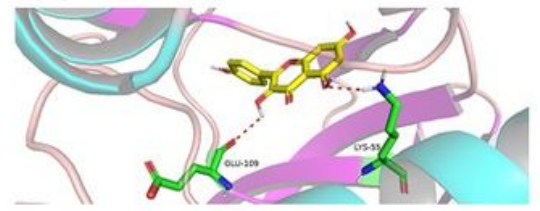

(h)

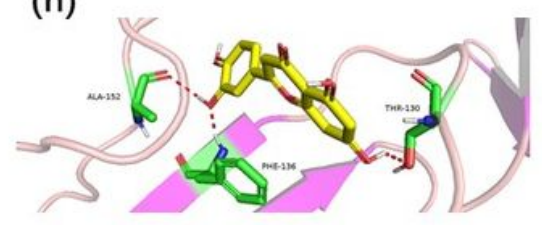

(c)

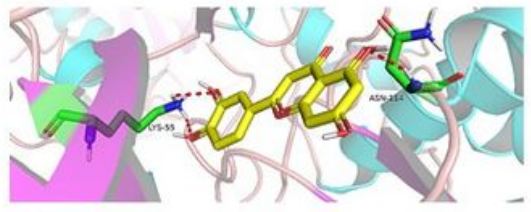

(f)

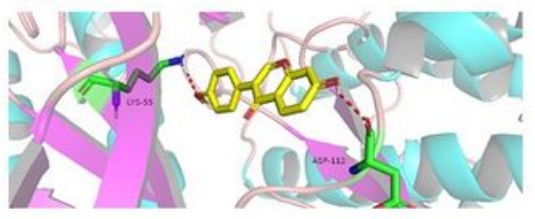

(i)

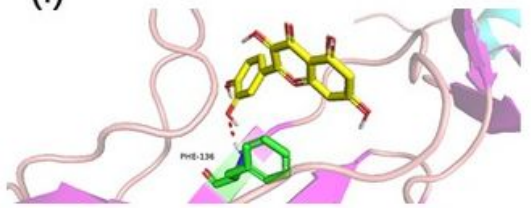




\section{Figure 8}

Analysis of target-compound docking simulation. (a) The site binding of quercetin and c-JUN. (b) The site binding of wogonin173 and c-JUN. (c) The site binding of luteolin and c-JUN. (d) The site binding of betasitosterol and c-JUN. (e) The site binding of kaempferol and c-JUN. (f) The site binding of formononetin and c-JUN. (g) The site binding of wogonin 173 and IL-6. (g) The site binding of luteolin and IL-6. (g) The site binding of quercetin and IL-6. 